Zum Bereich Informationsfreiheit sagte die Landesbeauftragte: „Auch wenn das zentrale elektronische Informationsregister auf der Seite www.bremen.de in den letzten Jahren sehr gewachsen ist (im Moment umfasst es um die 25.500 Dokumente), dürfen wir uns mit dem Erreichten nicht zufrieden geben. In einer lebendigen Verwaltung müssen die Informationsberge täglich ein gutes Stück wachsen. Wir haben noch nicht einmal die Baumgrenze erreicht. Und genauso wichtig ist es, dass auch die Zahl der Nutzerinnen und Nutzer noch einmal deutlich ansteigt. Amtliche Informationen wie die über die Bevorratung eines Grippemittels, leerstehende städtische Immobilien, die Folgekosten des Hafentunnels und über Kooperationen im Wissenschaftsbereich sind eine wichtige Voraussetzung dafür, bei den Entscheidungen, die uns alle betreffen, mitmischen zu können. Wenn es beim Zugang dazu noch hapert, helfen wir gerne mit."

Der 8. Jahresbericht schildert sieben Fälle, in denen sich Menschen aus Bremen an die Informationsfreiheitsbeauftragte gewandt haben, um sich Hilfe zu holen, weil öffentliche Stellen ihnen den Zugang zu öffentlichen Informationen verweigert hatten. In fünf Fällen war das schon erfolgreich. In den beiden weiteren Fällen sind die Diskussionen mit den betreffenden Stellen noch nicht abgeschlossen.

Hier kann der Bericht heruntergeladen werden: http://www.datenschutz-bremen.de/sixcms/media.php/13/36.+Jahresbericht+Datenschutz.pdf

\section{EuGH erklärt Richtlinie über die Vorratsdatenspeicherung für europarechtswidrig}

Die Bundesbeauftragte für den Datenschutz und die Informationsfreiheit, Andrea Voßhoff, begrüßt in einer PM das Urteil des EuGH als notwendige Klarstellung einer seit langem diskutierten Frage.

Andrea Voßhoff: „Die Entscheidung des Europäischen Gerichtshofes bringt nunmehr die dringend notwendige rechtliche Klarheit. Auch wenn das Gericht das Mittel der Vorratsdatenspeicherung nicht grundsätzlich als ungeeignet verworfen hat, hat es doch eindeutig entschieden, dass die Richtlinie Grenzen überschreitet, die zur Wahrung des Grundsatzes der Verhältnismäßigkeit eingehalten werden müssen. Das Urteil muss jetzt sorgfältig geprüft werden, auch mit Blick darauf, ob eine Neufassung der Richtlinie überhaupt sachgerecht ist. Ich halte es deshalb für geboten, jetzt erst einmal abzuwarten, bis der europäische Gesetzgeber sich entschieden hat, ob und wie eine europarechtskonforme Neuregelung der Richtlinie erfolgen soll."

Der Europäische Gerichtshof hat in seinem Urteil vom 08.04. 2014 die Richtlinie zur Vorratsdatenspeicherung (RL 2006/24/EG) rückwirkend für ungültig erklärt, da sie einen schwerwiegenden und unverhältnismäßigen Eingriff in die Grundrechte auf Achtung des Privatlebens und auf Schutz personenbezogener Daten und somit einen Verstoß gegen Artikel 7 und 8 der Charta der Grundrechte der Europäischen Union darstellt.

Gerichte aus verschiedenen Mitgliedstaaten hatten dem Europäischen Gerichtshof die Frage vorgelegt, ob die Richtlinie zur Vorratsdatenspeicherung mit den Vorschriften der europäischen Grundrechtecharta vereinbar sei. Bereits im Jahre 2010 hatte das Bundesverfassungsgericht die deutsche Umsetzung der Richtlinie für verfassungswidrig und nichtig erklärt; eine gesetzliche Neuregelung hat seither nicht stattgefunden.

\section{BSI: „Heartbleed Bug“ ist kritisch}

Der "Heartbleed-Bug", über den derzeit in den Medien berichtet wird, ist eine Sicherheitslücke in einer Programmerweiterung von OpenSSL namens "Heartbeat". OpenSSL ist eine freie Software-Bibliothek für Transport Layer Security (TLS) und umfasst Implementierungen verschiedener Verschlüsselungen. Insbesondere Webund Mail-Server aber auch andere Dienste wie Virtual Private Networks oder Appliances wie Router nutzen häufig diese Bibliothek für TLS/SSL-Verbindungen. Die Bibliothek enthält in den Versionen 1.0.1 bis 1.0.1f eine Schwachstelle, den "Heartbleed-Bug".

Das Bundesamt für Sicherheit in der Informationstechnik (BSI) stuft diese Schwachstelle als kritisch ein. Ein Angreifer ist unter Ausnutzung der Schwachstelle in der Lage, Speicherinhalte des OpenSSL Servers auszulesen, sofern diese die „Heartbeat"-Erweiterung aktiviert haben. Mithilfe des "Heartbleed Bugs“ können zudem unter Umständen die geheimen Schlüssel von OpenSSL-Servern ausgelesen werden.

Seit dem 7. April 2014 steht mit OpenSSL Version 1.0.1g ein Update zur Verfügung, das die Sicherheitslücke schließt. Betreiber, die auf ihren Servern OpenSSL einsetzen, sollten das Update umgehend einspielen. Falls seit März 2012 eine verwundbare OpenSSLVersion mit aktivierter Heartbeat-Erweiterung eingesetzt wurde, kann eine vergangene Kompromittierung von Schlüsseln nicht ausgeschlossen werden. Daher empfiehlt das BSI in einem solchen Fall den Austausch der verwendeten OpenSSL Server-, beziehungsweise Client-Zertifikate und Schlüssel sowie eine Änderung der verwendeten Passwörter. Der Austausch sollte erst nach der Einspielung des Updates erfolgen, da ansonsten die neuen Zertifikate wieder kompromittiert werden könnten. Die alten Zertifikate müssen nach erfolgreichem Austausch gesperrt werden. Betreiber sind aufgerufen, ihre Nutzer über die Umsetzung der Aktualisierung zu informieren, damit diese ihre Passwörter kurzfristig ändern.

\section{EDSB: Jahresbericht 2013}

Im Jahr 2013 war die Überarbeitung des EU-Datenschutzrahmens weiterhin ganz oben auf der Prioritätenliste des Europäischen Datenschutzbeauftragten (EDSB) in seiner Beratungstätigkeit zu neuen Rechtsetzungsvorhaben und wird auch 2014 eine Priorität bleiben. Weitere Schwerpunkte im letzten Jahr waren die Digitale Agenda und die Datenschutzrisiken neuer Technologien.

Die Umsetzung des Stockholm-Programms im Raum der Freiheit, der Sicherheit und des Rechts, Binnenmarktthemen wie die Reform des Finanzsektors, sowie Entwicklungen im Gesundheitswesen und bei Verbraucherthemen hatten ebenfalls Auswirkungen auf den Datenschutz. Der EDSB hat zusätzlich seine Zusammenarbeit mit anderen Aufsichtsbehörden ausgebaut, insbesondere im Hinblick auf IT-Großsysteme.

In der Aufsicht über die Verarbeitung personenbezogener Daten durch die Organe und Einrichtungen der EU hat der EDSB im Jahr 2013 mit mehr behördlichen Datenschutzbeauftragten in mehr Organen und Einrichtungen als je zuvor zusammengearbeitet. Zusätzlich haben mehrere Erhebungen des EDSB gezeigt, dass die meisten Organe und Einrichtungen der EU - viele Agenturen eingeschlossen - gute Fortschritte bei der Einhaltung der Datenschutzverordnung gemacht haben; nichtsdestotrotz sollten einige von ihnen ihre Anstrengungen verstärken.

Eine ausführliche Zusammenfassung des Jahresberichtes steht in deutscher Sprache zur Verfügung: https://secure.edps.europa.eu/ 